\title{
Investigation of Heterostructure Formed from Hole- and Electron-Doped Lanthanum Manganites
}

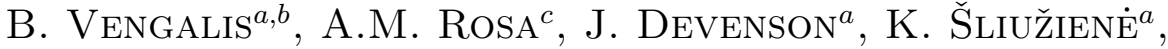 \\ V. Lisauskas ${ }^{a}$, A. $\operatorname{Oginskis}^{a}$, F. Anisimovas ${ }^{a}$ \\ AND V. PYRAGAS ${ }^{a}$ \\ ${ }^{a}$ Semiconductor Physics Institute, A. Goštauto 11, 01108 Vilnius, Lithuania \\ ${ }^{b}$ Vilnius Gediminas Technical University, Sauletekio al. 11
}

2040 Vilnius, Lithuania

${ }^{c}$ Dept. of Physics, University of Lisbon, Ed C8, 1749-016 Lisbon, Portugal

High crystalline quality films of $n$ - $\mathrm{La}_{2 / 3} \mathrm{Ce}_{1 / 3} \mathrm{MnO}_{3}, p$ - $\mathrm{La}_{2 / 3} \mathrm{Ca}_{1 / 3} \mathrm{MnO}_{3}$ and related $p-n$ diode structures were grown heteroepitaxially on latticematched $\mathrm{SrTiO}_{3}(100)$ substrates by dc magnetron sputtering and pulsed laser deposition. The $\mathrm{La}_{2 / 3} \mathrm{Ce}_{1 / 3} \mathrm{MnO}_{3} / \mathrm{La}_{2 / 3} \mathrm{Ca}_{1 / 3} \mathrm{MnO}_{3}$ bilayer was patterned into a strip-like geometry to investigate electrical properties of the interface. Significant magnetoresistance values and nonlinear current-voltage characteristics were indicated for the interface of the $p-n$ diode heterostructure.

PACS numbers: 73.40.Cg, 73.40.Lg, 75.50.Dd

\section{Introduction}

Hole-doped perovskite manganites referred to by general formula $\mathrm{Re}_{1-x} \mathrm{~A}_{x} \mathrm{MnO}_{3}(\mathrm{Re} \equiv \mathrm{La}, \mathrm{Nd}$; $\mathrm{A}$ is a divalent alkaline-earth element: $\mathrm{Ca}, \mathrm{Sr}, \mathrm{Ba})$ have been widely investigated in recent years due to their interesting properties such as transition from high resistance paramagnetic (PM) to a metallic ferromagnetic (FM) state, spin-polarized carriers below Curie temperature, $T_{\mathrm{c}}$, and colossal magnetoresistance (CMR) values measured in the vicinity of $T_{\mathrm{c}}$. [1]. Recently it has been found that electron-doped manganites exhibiting similar electrical and magnetic properties may be obtained via substituting $\mathrm{La}^{3+}$ by tetravalent cations such as Ce and $\mathrm{Sn}$ [2]. Thin films of electron-doped single-phase $\mathrm{La}_{1-x} \mathrm{Ce}_{x} \mathrm{MnO}_{3}$ (LCeMO) have been grown recently by pulsed laser deposition although a number of investigators pointed out difficulties of introducing Ce ions into $\mathrm{LaMnO}_{3}$ system in bulk samples. 
In this work, we report high quality films of electron-doped $\mathrm{La}_{2 / 3} \mathrm{Ce}_{1 / 3} \mathrm{MnO}_{3}$ and $p-\mathrm{La}_{2 / 3} \mathrm{Ca}_{1 / 3} \mathrm{MnO}_{3} / n-\mathrm{La}_{2 / 3} \mathrm{Ce}_{1 / 3} \mathrm{MnO}_{3}$ diode structures which are promising for various spintronics applications [3].

\section{Preparation and characterization of the films and heterostructures}

Thin films of $n$-type $\mathrm{La}_{2 / 3} \mathrm{Ce}_{1 / 3} \mathrm{MnO}$, hole-doped $\mathrm{La}_{2 / 3} \mathrm{Ca}_{1 / 3} \mathrm{MnO}_{3}$ (LCaMO) with a thickness ranging from 100 to $400 \mathrm{~nm}$, and the LCaMO/LCeMO diode structures were grown heteroepitaxially at $700-800^{\circ} \mathrm{C}$ on lattice-matched crystalline $\mathrm{SrTiO}_{3}(\mathrm{STO})(100)$ substrates by applying dc magnetron sputtering (MS) and pulsed laser deposition (PLD). Disk-shaped ceramic LCeMO and LCaMO targets $(3 \mathrm{~cm}$ in diameter) with $\mathrm{La} / \mathrm{Ce}$, and $\mathrm{La} / \mathrm{Ca}$ ratios $(3: 1)$ were used. After deposition, the films and heterostructures were saturated in oxygen followed by slow cooling down to room temperature. The crystalline structure of the prepared films and the LCaMO/LCeMO heterostructure were characterized by X-ray difraction (XRD) and reflected high-energy electron diffraction (RHEED).

The LCaMO/LCeMO bilayer was patterned in a cross-stripe geometry to investigate electrical properties of the prepared heterostructure. The underlying strip-like LCaMO film (200 $\mu \mathrm{m}$ in width) was patterned by applying a conventional lithography followed by chemical etching. The tape-like LCeMO film $(1 \mathrm{~mm}$ in width) was subsequently deposited by PLD at $750^{\circ} \mathrm{C}$ onto the patterned LCaMO layer through a mask. Low resistance electrodes used for electrical measurements were prepared by sputtering $\mathrm{Ag}$ onto the films and heterostructures. Electrical resistance, magnetoresistance, and voltage versus current were measured at $T=(78 \div 300) \mathrm{K}$.

\section{Results and discussion}

X-ray diffraction and reflected high energy electron diffraction investigations revealed epitaxial quality of the LCaMO, LCeMO films and the LCaMO/LCeMO bilayer structures when grown on lattice-matched $\mathrm{SrTiO}_{3}(a=0.3905 \mathrm{~nm})$. The off-plane lattice parameter of a pseudocubic unit cell of about $0.387 \mathrm{~nm}$ and $0.394 \mathrm{~nm}$ have been estimated from XRD measurements for the LCaMO, LCeMO films, respectively. Results concerning structure and electrical properties obtained in this work for LCaMO are in consistence with our earlier studies $[4,5]$.

In Fig. 1 we show resistance versus temperature for epitaxial LCeMO films of various thicknesses $(d \cong 200,300$, and $400 \mathrm{~nm})$ at $B=0$ (points) and $B=1.2 \mathrm{~T}$ (dotted lines) grown by PLD under the same deposition conditions $\left(T_{s}=750^{\circ} \mathrm{C}\right)$. Resistivity values ranging from about $3 \times 10^{-3}$ to $5 \times 10^{-3} \Omega \mathrm{cm}$ (at $T=300 \mathrm{~K}$ ) have been estimated for the films. The characteristic resistance peaks seen in the figure and a slight shift of the peak positions to higher temperatures with an applied magnetic field demonstrate clear evidence of paramagnetic to ferromagnetic transition. A slight increase in $T_{\mathrm{c}}$ seen in Fig. 1 with a film thickness decreasing is not clear as yet. Certainly, an increase in $T_{\mathrm{c}}$ with film 


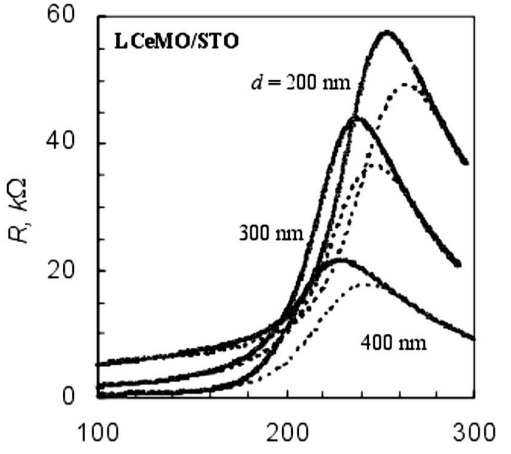

Fig. 1 $T, K$

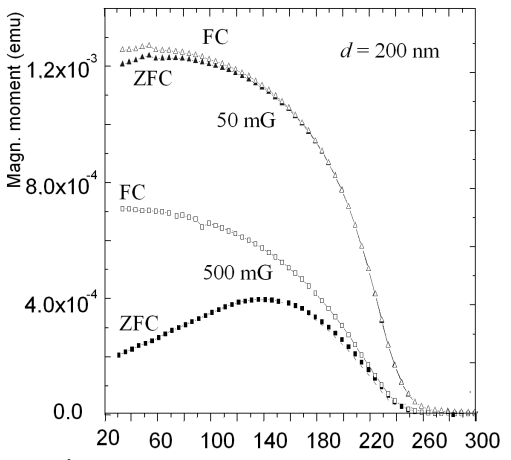

Fig. 2 $T(\mathrm{~K})$

Fig. 1. Resistance versus temperature of epitaxial $\mathrm{La}_{0.67} \mathrm{Ce}_{0.33} \mathrm{MnO}_{3} / \mathrm{SrTiO}_{3}$ films of various thicknesses $(d \cong 200,300$, and $400 \mathrm{~nm})$ measured at $B=0$ (points) and $1.2 \mathrm{~T}$ (dotted lines).

Fig. 2. Low-field magnetization $\left(B=50\right.$ and $500 \mathrm{mG}$ ) of $\mathrm{La}_{0.67} \mathrm{Ce}_{0.33} \mathrm{MnO}_{3} / \mathrm{SrTiO}_{3}$ film with a thickness $d \cong 200 \mathrm{~nm}$ measured in a field cooling (FC) and zero field cooling (ZFC) regimes.

thickness is usually observed either for Sr or Ca doped manganite films [4]. Figure 2 shows a typical magnetization versus temperature for the LCeMO/STO film $(d \cong 200 \mathrm{~nm})$. The measurements were carried out during film heating with an applied magnetic field $(B=50$ and $500 \mathrm{mG}$ ) and without magnetic field using a SQUID magnetometer.
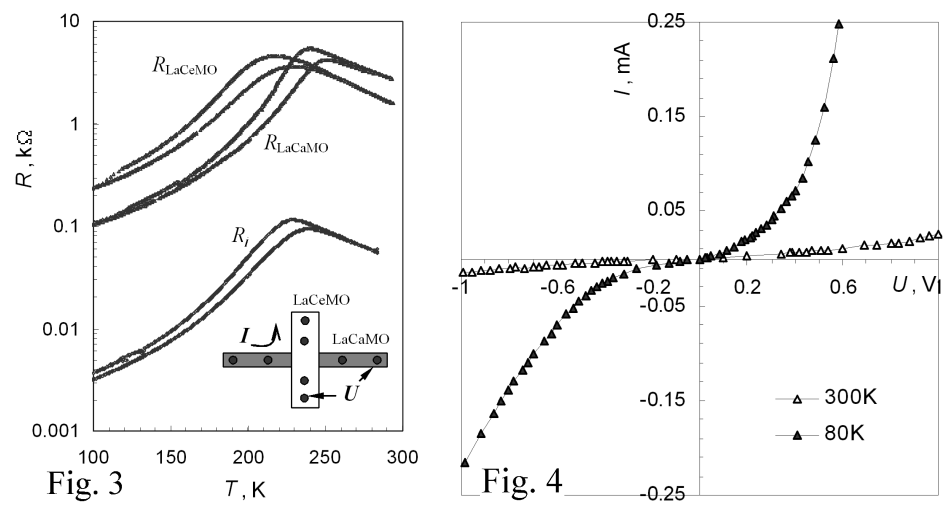

Fig. 3. Resistance versus temperature of the $\mathrm{La}_{0.66} \mathrm{Ca}_{0.34} \mathrm{MnO}_{3}$ and $\mathrm{La}_{0.66} \mathrm{Ce}_{0.34} \mathrm{MnO}_{3}$ films of the patterned bilayer structure and that of the $\mathrm{La}_{0.66} \mathrm{Ca}_{0.34} \mathrm{MnO}_{3} / \mathrm{La}_{0.66} \mathrm{Ce}_{0.34} \mathrm{MnO}_{3}$ interface. The sample used for vertical electrical transport (interface) investigation is shown in the inset.

Fig. 4. Current versus voltage of the $\mathrm{La}_{0.66} \mathrm{Ca}_{0.34} \mathrm{MnO}_{3} / \mathrm{La}_{0.66} \mathrm{Ce}_{0.34} \mathrm{MnO}_{3}$ interface measured at $300 \mathrm{~K}$ and $80 \mathrm{~K}$ for the LCaMO/LCeMO bilayer film structure patterned in cross-stripe geometry. 
Results of electrical measurements for the LCaMO/LCeMO bilayer structure patterned in cross-stripe geometry are presented in Fig. 3. This geometry allows one to study vertical electrical transport between the adjacent $n$ - and $p$-type manganite layers in order to investigate resistance of the interface, $R_{\mathrm{I}}$. It can be seen from this figure that both the LCaMO and LCeMO films and their interface exhibit a similar resistive transition at $235 \mathrm{~K}, 210 \mathrm{~K}$, and $215 \mathrm{~K}$, respectively. Both the intermediate position of the PM-FM transition temperature corresponding to the interface and the relatively low $R_{\mathrm{I}}$ values show the absence of high resistance interlayer and a possible oxygen outdiffusion from the interface. Furthermore, an increase in $R_{\mathrm{I}}$ with a magnetic field $\left(B=1.2 \mathrm{~T}\right.$ ) in the vicinity of $T_{\mathrm{c}}$ demonstrates a significant magnetoresistance of the $p-n$ manganite interface. Figure 4 demonstrates nonlinear $I-U$ characteristics of the LCaMO/LCeMO interface at $T=78$ and $300 \mathrm{~K}$ as well as asymmetry of the $I-U$ characteristics in respect to current direction which is typical of $p-n$ junctions.

Summarizing results of our investigations we conclude that electrical properties of the $\mathrm{La}_{2 / 3} \mathrm{Ce}_{1 / 3} \mathrm{MnO}_{3} / \mathrm{La}_{2 / 3} \mathrm{Ca}_{1 / 3} \mathrm{MnO}_{3}$ bilayer structure, namely, magnetoresistance and nonlinear electrical properties of the interface as well as the observed asymmetry of the $I-U$ characteristics may be promising for various applications.

\section{Acknowledgment}

This work was partially supported by CE project PRAMA.

\section{References}

[1] W.E. Pickett, J.S. Moodera, Physics Today 54, 39 (2001).

[2] T. Yanagida, T. Kanki, B. Vilquin, H. Tanaka, T. Kawai, Solid State Commun. 129, 785 (2004).

[3] C. Mitra, P. Raychaudhuri, K. Dörr, K.H. Müller, L. Schultz, P.M. Oppeneer, S. Wirth, Phys. Rev. Lett. 90, 017202 (2003).

[4] B. Vengalis, A. Maneikis, F. Anisimovas, R. Butkutè, L. Dapkus, A. Kindurys, J. Magn. Magn. Mater. 211, 35 (2000).

[5] B. Vengalis, F. Anisimovas, R. Butkute, L. Dapkus, A. Deksnys, V. Plaušinaitienè, A. Abrutis, A. Maneikis, J. Low Temp. Phys. 117, 1659 (1999). 